\title{
In vivo analysis of DNA methylation patterns recognized by specific proteins: coupling ChIP and bisulfite analysis
}

\author{
Maria Rosaria Matarazzo ${ }^{1}$, Francesca Lembo ${ }^{2}$, Tiziana Angrisano ${ }^{2}$, Esteban \\ Ballestar $^{3}$, Marcella Ferraro ${ }^{1}$, Raffaela Pero ${ }^{2}$, Maria Luigia De Bonis ${ }^{1}$, Carmelo \\ Bruno Bruni $^{2}$, Manel Esteller ${ }^{3}$, Maurizio D'Esposito ${ }^{1}$, and Lorenzo Chiariotti ${ }^{2,4}$ \\ ${ }^{1}$ Institute of Genetics and Biophysics, C.N.R., Naples, ${ }^{2}$ Università degli Studi di \\ Napoli, Naples, Italy, ${ }^{3}$ Spanish National Cancer Centre, Madrid, Spain, \\ and ${ }^{4}$ Università degli Studi di Catanzaro, Catanzaro, Italy
}

BioTechniques 37:666-673 (October 2004)

The three-way connection between DNA methylation, chromatin configuration, and transcriptional regulation is under increasing attention, but the fine rules governing the epigenetic control are still poorly understood. In several studies, the authors have concluded that the methylation status of $C p G$ sites could be critical for the binding of factors to DNA and, consequently, for chromatin conformation. We tested the possibility that a novel technical approach combining chromatin immunoprecipitation and bisulfite genomic sequencing analysis (ChIP-BA) could provide useful information on the role of specific $C p G$ methylation patterns in driving the association in vivo of proteins to given genomic regions. Our results show that ChIP-BA permits the establishment in vivo of the methylation patterns required for the binding of a methyl-CpG binding protein and, in addition, can potentially identify methylation patterns that do not allow a protein to bind specific genomic regions. Possible fields of application are discussed. We believe that wide use of ChIP-BA could make possible the exploration of a novel aspect of the intricate epigenetic web.

\section{INTRODUCTION}

Methylation of cytosines has a determinant role in genome plasticity and development. It may occur as a de novo and reversible event; the information stored in the distribution of 5-methylcytosine is flexible, stable, and heritable after cell division. Promoter methylation is often associated with transcriptional repression. This transcriptional control plays a pivotal role in mammalian development, X-inactivation, genomic imprinting, genome stability, and cancer (1). Defects in DNA methylation and/or in the interpretation of the methylation signal have been associated with a number of monogenic conditions (2) and, recently, with alterations of metabolism, such as the hyperhomocysteinemia (3) in humans. Methylation of $\mathrm{CpG}$ dinucleotides in gene regulatory regions has been proposed to trigger the binding of specific factors that recognize methylated $\mathrm{CpGs}$ and interpret the epigenetic signal possibly retained in both the density and the distribution of methyl-CpG sites.
These proteins are known as methyl$\mathrm{CpG}$ binding domain (MBD) proteins, and mammals have five well-known members, MeCP2, MBD1, MBD2, MBD3, and MBD4 (4). The best-established consequence of the binding of MBD proteins to a given promoter region is the recruitment of histonemodifying activities and chromatin-remodeling factors commonly resulting in transcriptional silencing (5). However, in other instances, a methyl binding protein, MBD2, can interact with other factors that favor transcriptional reactivation of methylated genes $(6,7)$.

All these observations strengthen the idea that MBD proteins are not simple mediators between DNA methylation and transcriptional repression but are fine interpreters of complex methylation signals. But, does a fine epigenetic code to be interpreted exist? Does the density or a specific arrangement of methylated $\mathrm{CpG}$ sites within a promoter region influence the binding ability and/or the functions of the MBD proteins and associated factors? Recently, some reports helped to shed some light on these questions (8-10). However, most of these works suffer the limitations of the in vitro approach.

Two in vivo techniques, such as chromatin immunoprecipitation (ChIP) and bisulfite genomic sequencing (BA), are largely used to study the profile of DNA methylation and the distribution of MBD proteins. Bisulfite genomic sequencing provides detailed information on the pattern of distribution of 5-methylcytosine along a genomic segment, on each individual strand (11). ChIP assays (12) have been used to identify in vivo the presence of MBD proteins in specific genomic region (13). Very recently, an approach that combines ChIP with methylation-sensitive restriction analysis has been used to analyze the methylation status of sequences bound by MBDs (14). Finally, the combination of ChIP assays with hybridization on a $\mathrm{CpG}$ island microarray (ChIP-on-chip analysis; Reference 15) has identified promoters targeted by specific transcription factors. Although these techniques have contributed to the understanding of the biology of MBD proteins, it remains unexplored whether differences in methylation profiles or densities in the same DNA regions may influence the binding in vivo of specific protein complexes.

Here we show that ChIP combined with bisulfite genomic analysis allows for the establishment in vivo of the methylation pattern of target DNA sequences selectively bound by a specific MBD protein. Most importantly, this novel technique has broader applicability since it can also be applied with many different antibodies raised against histone modifications that are now available. Therefore, this technique could also be applied to investigate the multiple connections between DNA methylation and histone modification patterns $(16,17)$, thus representing an important tool to decipher the interplay between different epigenetic signals.

\section{MATERIALS AND METHODS}

\section{Transfections and In Vitro Methylation}

Human embryonic kidney (HEK)293T cells were cultured in Dulbecco's modified Eagle's medium 
Table 1. Sequences of Amplification Primers

\begin{tabular}{|ll|}
\hline Primer & Sequence \\
\hline UMG1 & 5'-GCGCCAGGACCCTGAGGGAGGGGCTAGG-3' \\
UMG2 & 5'-TCCTGAGACCTGCTCCACCAGCAGTCAGAAGACTCC-3' \\
MG2 & 5'-TCCTAAAACCTACTCCACCAACAATCAAAAAACTCC-3' \\
PGK1-S & 5'-GTGTTAGGATTTTGAGGGAGGGTTAGG-3' \\
PGK1-AS & 5'-GAAGCCGCACGTCTCACTA-3' \\
PG-Forward & 5'-AGGAACAGGGCCCACACTAC-3' \\
PG-Reverse & 5'-GTTGTTTTGGGYGTGGTTTYGGGA-3' \\
SYB17 & 5'-ACTAAAAAAAAAAATCRATAATT-3' \\
SYB26 & 5'-CCAAGAGGCCACGCGTAG-3' \\
\#1 & 5'-GTTCTCTCCCCGCCTCCC-3' \\
\#2 & 5'-ATGATTTTGATTTGTTTTTTTT-3' \\
\#3 & 5'-ATTTAACTCTCTTTCAATTATA-3' \\
\#4 & 5'-GTAGGAGAGTAAAAGGATTTAG-3' \\
\hline
\end{tabular}

(DMEM) supplemented with $10 \%$ fetal calf serum (FCS; Invitrogen, Carlsbad, CA, USA). Cells were plated at a density of about 250,000 per 60 -mm Petri ${ }^{\circledR}$ dish $16 \mathrm{~h}$ before transfections. DNA transfections (2-5 $\mu \mathrm{g}$ purified plasmids; Qiagen, Valencia, CA, USA) were carried out by calcium phosphate precipitation using CalPhos ${ }^{\mathrm{TM}}$ Mammalian Transfection Kit (BD Biosciences Clontech, San Jose, CA, USA) according to the manufacturer's instructions.

In vitro methylation was performed as follows: $50 \mu \mathrm{g}$ of pGAT1700 plasmid were treated with $50 \mathrm{U}$ of $S s s \mathrm{I}$ methylase (New England Biolabs, Beverly, MA, USA) at $37^{\circ} \mathrm{C}$ in the presence of $5 \mathrm{mM}$ adenosylmethionine for $8 \mathrm{~h}$.

\section{Chromatin Immunoprecipitation}

ChIP experiments were performed on entire cells according to the method of Orlando et al. (12), with some modifications. Forty-eight hours after transfection, $0.5-2 \times 10^{6}$ cells per immunoprecipitation were treated with $1 \%$ formaldehyde for $10 \mathrm{~min}$ at $37^{\circ} \mathrm{C}$. Ultrasound sonication was performed under conditions that gave a range in DNA fragments from 200-1000 bp. After a centrifugation step (at 16,100x $g$ ) to reduce debris, the soluble chromatin was diluted with ChIP dilution buffer $[0.01 \%$ sodium dodecyl sulfate (SDS), $1.1 \%$ Triton ${ }^{\circledR} \mathrm{X}-100,1.2 \mathrm{mM}$ EDTA, 16.7 mM Tris-HCl, pH 8.1, $167 \mathrm{mM} \mathrm{NaCl}$, and protease inhibitors (100 mM PMSF and aprotinin, both from Sigma, St. Louis, MO, USA)]. Immunoprecipitations of cross-linked complexes were performed using 10 $\mu \mathrm{g}$ of mouse monoclonal anti-myc antibody or anti-MeCP2 antibody (Santa Cruz Biotechnology, Santa Cruz, CA, USA). For each experiment, a sample without antibody was carried out in parallel as a control for nonspecific background. After an overnight incubation at $4^{\circ} \mathrm{C}$, the bound chromatin-antibody complexes were brought down to room temperature with Protein A Sepharose ${ }^{\mathrm{TM}}$ CL-4B beads (Amersham Biosciences, Piscataway, NJ, USA). The supernatant fraction was kept as the unbound DNA control. Bound and unbound samples were then incubated at $65^{\circ} \mathrm{C}$ to reverse protein-DNA crosslinks. Phenol-chloroform extraction and ethanol precipitation allowed for the purification of DNA, which was successively UV quantified and used for PCR (50 ng). To ensure linearity of amplification, different cycle numbers were tested (data not shown). PCRs were then performed using Ampli$\mathrm{Taq}^{\circledR}$ (Roche Applied Science, Indianapolis, IN, USA) for 1 cycle of $95^{\circ} \mathrm{C}$ for $2 \mathrm{~min}$ and 20 cycles of $95^{\circ} \mathrm{C}$ for 1 $\min , 55^{\circ} \mathrm{C}$ for $1 \mathrm{~min}$, and $72^{\circ} \mathrm{C}$ for 1 min (galectin- 1 ); 1 cycle of $95^{\circ} \mathrm{C}$ for 3 min; 30 cycles of $95^{\circ} \mathrm{C}$ for $30 \mathrm{~s}, 60^{\circ} \mathrm{C}$ for $45 \mathrm{~s}$, and $72^{\circ} \mathrm{C}$ for $45 \mathrm{~s}(P G K 1$ and $S Y B L 1)$. Primers used were UMG1 and UMG2 (galectin-1); PGK1-S and PGK1-AS (PGK1), and SYB17 and SYB26 (SYBL1). All primer sequnces are listed in Table 1. 


\section{Bisulfite Genomic Sequencing}

The bisulfite reaction was carried out using $2 \mu \mathrm{g}$ of MBD2-bound or MeCP2-bound DNA, according to a previously described protocol (11), with some modifications. We did not perform enzymatic digestion of DNA because the DNA was sonicated before immunoprecipitation. The DNA was denatured by adding freshly prepared $\mathrm{NaOH}$ to a final concentration of $0.3 \mathrm{M}$ and incubated for $15 \mathrm{~min}$ at $50^{\circ} \mathrm{C}$. Hydroquinone $(75 \mathrm{mM})$ and $3.5 \mathrm{M}$ sodium bisulfite (Sigma), pH 5.0 (pH adjustment with $10 \mathrm{M} \mathrm{NaOH}$ ), were freshly prepared before use. Final concentrations of $3.1 \mathrm{M}$ sodium bisulfite and 0.5 $\mathrm{mM}$ hydroquinone and $10 \mu \mathrm{g}$ of glycogen were then added to denatured DNA in a final reaction volume of $250 \mu \mathrm{L}$. The samples were gently mixed, overlaid with mineral oil, and incubated at $50^{\circ} \mathrm{C}$ for $16 \mathrm{~h}$. The DNA was desalted and concentrated using Geneclean ${ }^{\circledR}$

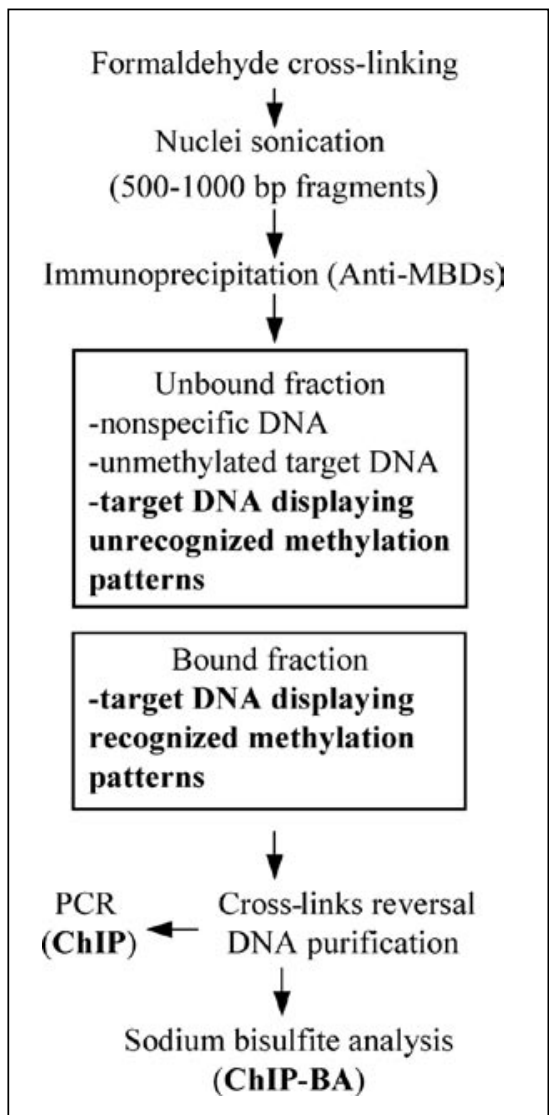

Figure 1. Flowchart of the chromatin immunoprecipitation bisulfite analysis (ChIP-BA) procedure. $\mathrm{MBDs}$, methyl-CpG binding domain proteins.
(Qbiogene, Carlsbad, CA, USA), denatured with $0.3 \mathrm{M} \mathrm{NaOH}$ for $15 \mathrm{~min}$ at $37^{\circ} \mathrm{C}$, neutralized with $3 \mathrm{M}$ ammonium acetate, $\mathrm{pH}$ 7.0, and precipitated with ethanol and $10 \mu \mathrm{g}$ of glycogen. The DNA was resuspended in $50 \mu \mathrm{L}$ of deionized water. We then performed PCR amplification on $5 \mu \mathrm{L}$ (about $50 \mathrm{ng}$ ) of the sodium bisulfite-treated DNA samples using modified primers. All PCRs were carried out in $100 \mu \mathrm{L}$ volumes containing $10 \mathrm{mM}$ Tris, $50 \mathrm{mM} \mathrm{KCl}$, $1 \mathrm{mM} \mathrm{MgCl} ; 2 \%$ dimethyl sulfoxide (DMSO), $0.2 \mathrm{mM}$ dNTPs, $10 \mathrm{pmol}$ of each primer, and $2 \mathrm{U}$ of $\mathrm{Taq}$ DNA polymerase (AmpliTaq). Primer used were MG1 and MG2 (galectin-1), PGforward and PG-reverse $(P G K 1)$, and $\# 1, \# 2, \# 3$ and \#4 (SYBL1). All primer sequences are shown in Table 1 . The amplification cycles were as previously described (6). The amplified fragments were cloned into the pGEM $^{\circledR}$-T Easy Vector (Promega, Madison, WI, USA), and then 20 independent clones for each fragment were sequenced to determine the methylation pattern of individual DNA.

\section{RESULTS AND DISCUSSION}

In order to devise a method that could establish whether differences in methylation profiles or densities in the same DNA regions may influence the binding in vivo of specific protein complexes, we decided to combine the ChIP and bisulfite genomic sequencing techniques (ChIP-BA). The approach consists of the bisulfite analysis of the methylation profile of specific DNA fragments immu-

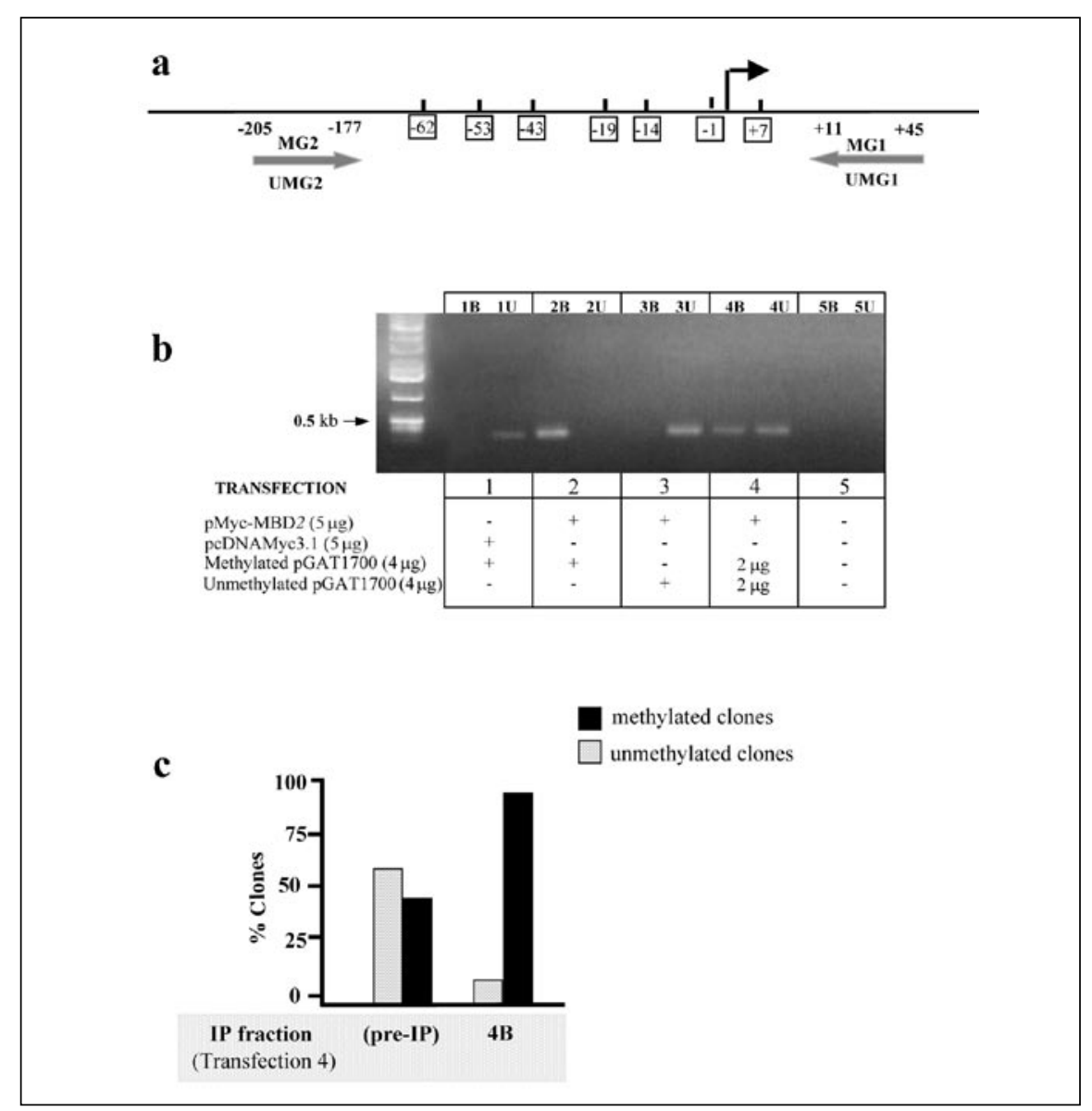

Figure 2. Chromatin immunoprecipitation bisulfite analysis (ChIP-BA) on transfected genes. (a) Map of $\mathrm{CpG}$ sites and primer positions, relative to transcription initiation site, in the transfected galectin1 promoter. (b) ChIP showing in vivo interaction of MBD2 with the methylated pGAT1700 plasmid. B, bound fraction; U, unbound fraction. Data obtained by sequencing SP6 primer with at least 20 plasmid clones for each sample were expressed as the ratio of clones showing methylated (or unmethylated) profiles to the number of clones. Pre-IP, whole cell lysate from transfection 4 before immunoprecipitation; 4B, MBD2-bound fraction from transfection 4 (see text). 
noprecipitated with antibodies against different methyl-binding proteins. The main steps of ChIP-BA are outlined in Figure 1. To evaluate the potential applicability of ChIP-BA methodology, we tested this technique on three different experimental systems to analyze either transfected or endogenous genes and target sequences.

First, ChIP-BA was performed by cotransfecting cultured cells with both methylated target sequences and an expression vector for the methyl-CpG binding protein MBD2. As target DNA, we used the pGAT1700 plasmid, which contains $1700 \mathrm{bp}$ of the mouse galectin-1 gene. The region from -177 to +11 contains 7 methylatable $\mathrm{CpG}$ sites (Figure 2a) that, when methylated, induce transcriptional silencing (18) mediated by MBD2 (6). HEK293T cells were cotransfected with pMyc-MBD2 along with either in vitro methylated or unmethylated pGAT1700 (Figure 2b, bottom). To immunoprecipitate MycMBD2/DNA complexes, we performed ChIP using anti-myc antibody. We then performed PCR to amplify the mouse galectin-1 promoter. The results presented in Figure 2b show a specific interaction of MBD2 with the methylated form of the pGAT1700 plasmid, as revealed by the strong enrichment of the amplification signal in the bound fraction (lane 2B) compared to the unbound fraction (lane 2U). Conversely, when unmethylated pGAT1700 plasmid was transfected along with MBD2 (transfection 3) or when pcDNAMyc3.1 empty vector was transfected along with the methylated pGAT1700 plasmid (transfection 1), the fragment was amplified in unbound (lanes $3 \mathrm{U}$ and $1 \mathrm{U}$ ) but not in bound fractions (lane $3 \mathrm{~B}$ and $1 \mathrm{~B})$. Finally, when both methylated and unmethylated pGAT1700 were transfected (transfection 4), the amplification signal was present in both the bound and the unbound fractions (lanes $4 \mathrm{U}$ and $4 \mathrm{~B}$ ). No signal was detected in untransfected cells (lanes 5B and 5U), confirming the specificity of the used oligonucleotides for the transfected mouse galectin-1 promoter.

To analyze the methylation pattern of transfected pGAT1700 at high resolution, we performed sodium bisulfite methylation analysis on the immunoprecipitated DNA (Figure 2c). The bi- sulfite reaction was carried out using 2 $\mu \mathrm{g}$ of MBD2-bound DNA (fraction 4B) and $2 \mu \mathrm{g}$ of DNA from transfection 4 before immunoprecipitation. Figure $2 \mathrm{c}$ shows the percentage of methylated and unmethylated clones found in each DNA fraction. We found a very strong enrichment of the methylated plasmid in the immunoprecipitated fraction (4B) compared to DNA analyzed before immunoprecipitation.

Because the transfected plasmids are fully methylated, the methylation of the vector backbone could influence the chromatin structure and affect the binding of MBD proteins. We therefore applied the ChIP-BA procedure to analyze endogenous genes.

The ChIP-BA assay was tested in a model system in which we used femalederived lymphoblastoid cell lines to analyze an endogenous gene, the X-linked $P G K 1$. In females, two different methylation statuses coexist for this gene, with the inactive allele methylated and the active one unmethylated (19). In fact, when we performed direct bisulfite sequencing of $P G K 1$ with total DNA, we found that both methylated and unmethylated CpGs were present (Figure 3A). We then performed ChIP assays with anti-MeCP2 antibodies; the immunoprecipitated DNA was subjected to bisulfite sequencing prior to PCR amplification. In this case, only the methylated sequence was found (Figure 3A). These results confirmed the suitability of this technique and indicated that MeCP2 is only bound to the promoter of the $P G K 1$ gene when methylated.

Finally, we analyzed the methylation patterns of the promoter region of synaptobrevin-like 1 gene (SYBL1), present in both human X- and Y-chromosomes. SYBL1 is subject to monoallelic inactivation on Y- and inactive $\mathrm{X}$-chromosomes through the methylation of a 5' $\mathrm{CpG}$ island (20) that spans the region from nucleotides -252 to +227, including $37 \mathrm{CpG}$ dinucleotides (Figure 3B). This region shows complete absence of methylation on the expressed alleles of male and active female X-chromosomes, whereas both the repressed alleles of the Y- and inactive X-chromosomes are widely methylated, hypoacetylated (21), and bound in vivo by MBDs (M.R. Matarazzo, M.L. De Bonis, and M. D'Esposito, unpublished data). We used a lymphoblastoid cell line derived from an adult male $(\mathrm{AB})$, which was heterozygous for a polymorphic site in the promoter of the gene, allowing for the SYBL1 X and $\mathrm{Y}$ alleles to be distinguished (Figure $3 \mathrm{~B}$ ). By using this additional tool, since the polymorphic nucleotide was preserved in the antisense DNA strand following bisulfite treatment (21), we could discriminate the allele-specific methylation patterns of cloned DNA fragments after the ChIP-BA procedure. Then, we easily compared only the patterns corresponding to the $\mathrm{Y}$ alleles derived from the different fractions of chromatin. After ChIP, using anti-MeCP2 antibody (M.R. Matarazzo, M.L. De Bonis, and M. D'Esposito, unpublished data), MeCP2-bound DNA and input DNA were modified with sodium bisulfite, and the PCR products were obtained through two rounds of PCR using a nested primers approach. Fifteen clones each for the input and for MeCP2-bound DNA were sequenced. As expected, roughly fifty percent of the clones from the input fraction corresponded to the $\mathrm{Y}$ allele, whereas in the MeCP2-bound fraction, there were only $\mathrm{Y}$ alleles. The methylation profiles relative to these alleles were represented in a box plot graphic (Figure 3B). A comparison between the methylation level of the SYBL1 CpG island of the Y alleles found in the MeCP2 immunoprecipitated fraction and that found in the input fraction showed that more densely methylated fragments were enriched in the MeCP2-bound fraction. This was not the case when ChIP experiments were performed on the $S Y B L 1$ promoter by using antibodies against MBD2 (data not shown), a protein that displays the ability to bind in vitro both high and low methylated DNA (4). These data confirm that by using the ChIP-BA technique, it is possible to determine the methylation status required in vivo for $\mathrm{MeCP} 2$ to bind a specific sequence.

The technique described here, ChIPBA, can potentially identify specific patterns or specific $\mathrm{CpG}$ sites whose methylation status is determinant for the in vivo binding or the exclusion of any methyl-binding protein. It is also clear that ChIP-BA could be used successfully to establish the methylation patterns that influence the binding 
ability in vivo of other proteins such as transcription factors. Moreover, by using antibodies raised against different modifications of histones, it might be possible to investigate the correlation between specific methylation patterns, histone modifications, and the local chromatin configuration. In fact, several crucial studies are currently investigating the methylation or acetylation of histones in correlation with local DNA methylation status $(16,17)$, and the precise timing of these events is also under increasing attention to define the interplay between these events. ChIP-BA can find useful application in
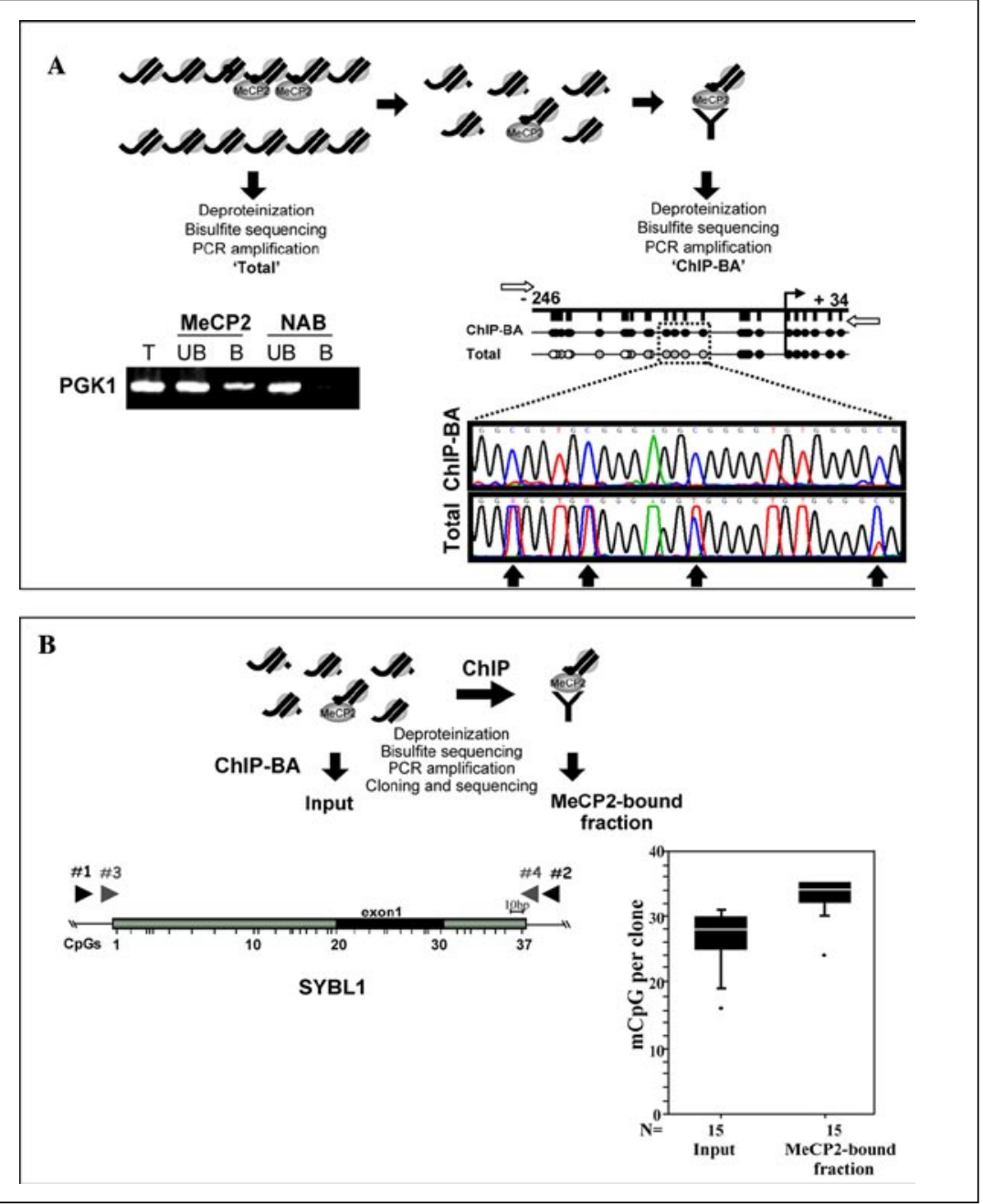

Figure 3. Chromatin immunoprecipitation bisulfite analysis (ChIP-BA) on endogenous genes. (A) Top: scheme of ChIP-BA assay for the X-linked PGK1 gene. Bottom left: ChIP assay on PGK1 promoter using anti-MeCP2 antibodies. A no antibody control (NAB) is also shown. T, pre-IP fraction; UB, unbound fraction; $\mathrm{B}$, bound fraction. The bands represent the amplified PGK1 promoter fragments from the indicated fractions. Bottom right, representation of some of the $\mathrm{CpG}$ sites (black bars) included in the PCR fragment. Primers used (empty arrows) were PG-forward and PG-reverse (Table 1). Methylation status of CpG sites: black ( $100 \%$ methylation), gray ( $>20 \%$ methylation), and white ( $<20 \%$ methylation). An indicative sequence analysis of part of the fragment is shown for both total DNA (total) and chromatin-immunoprecipitated DNA (ChIP-BA). Black arrows indicate the CpGs that are fully methylated in the ChIP-BA sample. (B) Top: scheme of the ChIP-BA assay for SYBL1 gene methylation analysis. Bottom left: map of the SYBL1 CpG island containing $37 \mathrm{CpG}$ dinucleotides, from position -252 to +227 . Exon 1 is indicated as a black box. Primer positions (\#1, \#2,\#3, and \#4) are indicated. Bottom right: summary of the bisulfite methylation analysis of SYBL1 CpG island Y alleles. MeCP2-bound DNA is compared to the input fraction. The numbers of $\mathrm{mCpG}$ per clone for each fraction are represented in box plots. Boxes correspond to the distribution of $\mathrm{mCpGs}$ per clone in the interquartile range (containing $50 \%$ of the values), and the end of vertical lines or "whiskers" indicate the highest and lowest values. The presence of outliers is underlined with points outside the ends of the whiskers. The median is indicated by a line across the box. The number of clones $(\mathrm{N})$ analyzed for each sample is reported on the abscissa. Pre-IP, whole cell lysate before immunoprecipitation.

several fields of studies involving DNA methylation analysis, such as imprinting, $\mathrm{X}$ inactivation, development, cancer, and genetic diseases. We believe that wide use of ChIP-BA may offer a substantial contribution to demonstrate the existence of an epigenetic code, and eventually, to gain insights into the logic of epigenetic signals and their interpretation.

\section{ACKNOWLEDGMENTS}

M.R.M., F.L., and T.A. contributed equally to this work. This work was supported by grants from the Associazione Italiana per la Ricerca sul Cancro (AIRC) (to L.C.), Ministero dell'Università e della Ricerca (MIUR) (to L.C. and C.B.B.), Ministero della Salute (to L.C.), and by Telethon grant no. GGP02308 and MIUR cluster 02 (to M.D.E.). M.E.'s laboratory is funded by a SAF2001-0059 MCYT grant and by the International Rett Syndrome Association. E.B. is funded by the Ramon y Cajal MCYT programme.

\section{COMPETING INTERESTS STATEMENT}

The authors declare no competing interests.

\section{REFERENCES}

1.Robertson, K.D. and A.P. Wolffe. 2000. DNA methylation in health and disease. Nat. Rev. Genet. 1:11-19.

2.Hendrich, B. and W. Bickmore. 2001. Human diseases with underlying defects in chromatin structure and modification. Hum. Mol. Genet. 10:2233-2242.

3.Ingrosso, D., A. Cimmino, A.F. Perna, L. Masella, N.G. De Santo, M.L. De Bonis, M. Vacca, M. D'Esposito, et al. 2003. Folate treatment reverts unbalanced methylation and alterations of allelic expression in hyperhomocysteinemic/uremic patients. Lancet 361:1693-1699.

4.Hendrich, B. and S. Tweedie. 2003. The methyl-CpG binding domain and the evolving role of DNA methylation in animals. Trends Genet. 19:269-277.

5.Ballestar, E. and A.P. Wolffe. 2001. MethylCpG-binding proteins. Targeting specific gene repression. Eur. J. Biochem. 268:1-6.

6.Lembo, F., R. Pero, T. Angrisano, C. Vitiello, R. Iuliano, C.B. Bruni, and L. Chiariotti. 2003. MBDin, a novel MBD2-interacting protein, relieves MBD2 repression potential 
and reactivates transcription from methylated promoters. Mol. Cell. Biol. 23:1656-1665.

7.Hutchins, A.S., A.C. Mullen, H.W. Lee, K.J. Sykes, F.A. High, B.D. Hendrich, A.P. Bird, and S.L. Reiner. 2002. Gene silencing quantitatively controls the function of a developmental trans-activator. Mol. Cell 10:81-91.

8.Fraga, M.F., E. Ballestar, G. Montoya, P. Taysavang, P.A. Wade, and M. Esteller. 2003. The affinity of different MBD proteins for a specific methylated locus depends on their intrinsic binding properties. Nucleic Acids Res. 31:1765-1774.

9.Ballestar, E., T.M. Yusufzai, and A.P. Wolffe. 2000. Effects of Rett syndrome mutations of the methyl-CpG binding domain of the transcriptional repressor $\mathrm{MeCP} 2$ on selectivity for association with methylated DNA. Biochemistry 39:7100-7106.

10.Kudo, S., Y. Nomura, M. Segawa, N. Fujita, M. Nakao, J. Dragich, C. Schanen, and M. Tamura. 2001. Functional analyses of $\mathrm{MeCP} 2$ mutations associated with Rett syndrome using transient expression systems. Brain Dev. 23(Suppl 1):S165-173.

11.Frommer, M., L.E. McDonald, D.S. Millar, C.M. Collis, F. Watt, G.W. Grigg, P.L. Molloy, and C.L. Paul. 1992. A genomic sequencing protocol that yields a positive display of 5-methylcytosine residues in individual DNA strands. Proc. Natl. Acad. Sci. USA 89:1827-1831.

12.Orlando, V., H. Strutt, and R. Paro. 1997. Analysis of chromatin structure by in vivo formaldehyde cross-linking. Methods 11:205214

13.Fournier, C., Y. Goto, E. Ballestar, K. Delaval, A.M. Hever, M. Esteller, and R. Feil. 2002. Allele-specific histone lysine methylation marks regulatory regions at imprinted mouse genes. EMBO J. 21:6560-6570.

14.Ghoshal, K., S. Majumder, J. Datta, T. Motiwala, S. Bai, S.M. Sharma, W. Frankel, and S.T. Jacob. 2004. Role of human ribosomal RNA (rRNA) promoter methylation and of methyl-CpG-binding protein MBD2 in the suppression of rRNA gene expression. J. Biol. Chem. 279:6783-6793.

15.Ballestar, E., M.F. Paz, L. Valle, S. Wei, M.F. Fraga, J. Espada, J.C. Cigudosa, T.H. Huang, et al. 2003. Methyl-CpG binding proteins identify novel sites of epigenetic inactivation in human cancer. EMBO J. 22:63356345.

16.Tariq, M., H. Saze, A.V. Probst, J. Lichota, Y. Habu, and J. Paszkowski. 2003. Erasure of $\mathrm{CpG}$ methylation in Arabidopsis alters patterns of histone $\mathrm{H} 3$ methylation in heterochromatin. Proc. Natl. Acad. Sci. USA 100:88238827.

17.Gregory, R.I., T.E. Randall, C.A. Johnson, S. Khosla, I. Hatada, L.P. O'Neill, B.M. Turner, and R. Feil. 2001. DNA methylation is linked to deacetylation of histone H3, but not H4, on the imprinted genes Snrpn and U2af1-rs1. Mol. Cell. Biol. 21:5426-5436.

18.Benvenuto, G., M.L. Carpentieri, P. Salvatore, L. Cindolo, C.B. Bruni, and L. Chiariotti. 1996. Cell-specific transcriptional regulation and reactivation of galectin-1 gene expression are controlled by DNA methylation of the promoter region. Mol. Cell. Biol.
16:2736-2743

19.Pfeifer, G.P., R.L. Tanguay, S.D. Steigerwald, and A.D. Riggs. 1990. In vivo footprint and methylation analysis by PCR-aided genomic sequencing: comparison of active and inactive $\mathrm{X}$ chromosomal DNA at the $\mathrm{CpG}$ island and promoter of human PGK-1. Genes Dev. 4:1277-1287.

20.Huber, R., R.S. Hansen, M. Strazzullo, G. Pengue, R. Mazzarella, M. D'Urso, D. Schlessinger, G. Pilia, et al. 1999. DNA methylation in transcriptional repression of two differentially expressed X-linked genes, GPC3 and SYBL1. Proc. Natl. Acad. Sci. USA 96:616621.

21.Matarazzo, M.R., M.L. De Bonis, R.I. Gregory, M. Vacca, R.S. Hansen, G. Mercadante, M. D'Urso, R. Feil, et al. 2002. Allelic inactivation of the pseudoautosomal gene SYBL1 is controlled by epigenetic mechanisms common to the $\mathrm{X}$ and $\mathrm{Y}$ chromosomes. Hum. Mol. Genet. 11:3191-3198.

Received 26 March 2004; accepted 7 June 2004.

Address correspondence to Maurizio D'Esposito, Institute of Genetics and Biophysics, via Marconi 10, 80125 Naples, Italy,e-mail: desposit@iigb.na.cnr.it or to L. Chiariotti, Dipartimento di Biologia e Patologia Cellulare e Molecolare, via S. Pansini, 5, 80131 Naples, Italy, e-mail: chiariot@unina.it 